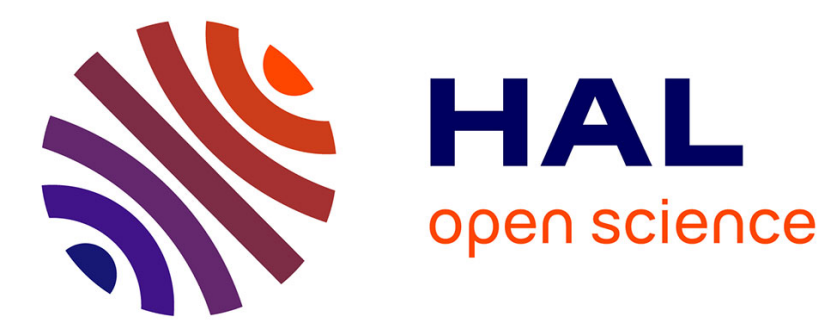

\title{
Pseudoelastic Shape Memory Alloys to Mitigate the Flutter Instability: A Numerical Study
}

\author{
Arnaud Malher, Olivier Doaré, Cyril Touzé
}

\section{To cite this version:}

Arnaud Malher, Olivier Doaré, Cyril Touzé. Pseudoelastic Shape Memory Alloys to Mitigate the Flutter Instability: A Numerical Study. M. Belhaq. Structural Nonlinear Dynamics and Diagnosis, 168, Springer, pp.353-365, 2015, Springer Proceedings in Physics, 978-3-319-19851-4. 10.1007/978-3319-19851-4_17. hal-01206679

\section{HAL Id: hal-01206679 \\ https://hal-ensta-paris.archives-ouvertes.fr/hal-01206679}

Submitted on 17 Nov 2017

HAL is a multi-disciplinary open access archive for the deposit and dissemination of scientific research documents, whether they are published or not. The documents may come from teaching and research institutions in France or abroad, or from public or private research centers.
L'archive ouverte pluridisciplinaire HAL, est destinée au dépôt et à la diffusion de documents scientifiques de niveau recherche, publiés ou non, émanant des établissements d'enseignement et de recherche français ou étrangers, des laboratoires publics ou privés. 


\title{
Pseudoelastic Shape Memory Alloys to Mitigate the Flutter Instability: A Numerical Study
}

\author{
Arnaud Malher, Olivier Doaré and Cyril Touzé
}

\begin{abstract}
A passive control of aeroelastic instabilities on a two-degrees-of-freedom (dofs) system is considered here using shape memory alloys (SMA) springs in their pseudo-elastic regime. SMA present a solid-solid phase change that allow them to face strong deformations $(\sim 10 \%)$; in the pseudo-elastic regime, an hysteresis loop appears in the stress-strain relationship which in turn gives rise to an important amount of dissipated energy. This property makes the SMA a natural candidate for mitigating undesired vibrations in a passive manner. A 2-dofs system is used here to model the classical flutter instability of a wing section in a uniform flow. The SMA spring is selected to act on the pitch in order to dissipate energy of the predominant motion. A simple phenomenological model for the SMA hysteresis loop is introduced, allowing for a quantitative study of the important parameters to optimize in view of an experimental design. Thanks to a simple phenomenological model for the SMA hysteresis loop, a quantitative numerical study is performed in order to exhibit the best tuning of the material parameters for controlling the flutter instability.
\end{abstract}

\section{Introduction}

Aeroelastic instabilities are an important issue in aeronautics, especially regarding the wing motions. Indeed, for a coupled system airflow-flexible structure, like aircraft wing, turbojet or bridge, a limit velocity exists above which the flexible structure cannot evacuate the energy received from the airflow anymore, then giving rise to strong or even fatal deformations. These instabilities, usually described under the generic term flutter instability, result from interaction between aerodynamic, inertia and elastic forces [3]. In this contribution, we focus on a passive control device for mitigating the flutter instability by using springs composed of shape memory alloys (SMA). In their pseudo-elastic regime, SMA are known for showing the ability of

\footnotetext{
A. Malher · O. Doaré · C. Touzé

Unité de Mécanique (UME), ENSTA-ParisTech, 828 Boulevard des Maréchaux,

91762 Palaiseau Cedex, France

e-mail: arnaud.malher@ensta-paristech.fr

O. Doaré

e-mail: olivier.doare@ensta-paristech.fr

C. Touzé

e-mail: cyril.touze@ensta-paristech.fr
} 
dissipating an important amount of energy thanks to the hysteresis loop appearing in their stress-strain relationship [11], and has thus already been used in numerous applications ranging from civil engineering, aeronautics to medical industry [13, 14]. The goal of this study is, in a first step, not to describe the system as accurately as possible but to exhibit the interest of using an hysteretic phenomenon in the view of controlling an aeroelastic instability and studying the influence of different SMA parameters for the flutter control.

Recent contributions have considered the dynamical responses of SMA springs from the theoretical viewpoint $[1,7,11]$ in order to properly quantify the most prominent features of the vibrations of simple single dof systems. Experimentally, a torsion pendulum has recently been used in order to clearly exhibit the softening effect of SMA oscillators [4].

The aim of this paper is to investigate the effect of a SMA spring on the flutter instability. More particularly, the most relevant parameters of a pseudo-elastic regime on the amplitudes of the limit cycle oscillations (LCO) are analyzed, in order to quantify the effect of the dissipation brought by the hysteresis loop. The airfoil is modeled using the classical 2-dofs system coupling pitch and heave motions [5]. In order to exchange energy and create the possibility of a Hopf bifurcation in the system, the minimal model should contain at least a flexural (heave) and a torsional (pitch) mode. The SMA nonlinear behaviour is described by an heuristic model where the prominent parameters are left free to vary. The structural nonlinearities that may appear in the wing motion are here described thanks to the addition of a cubic nonlinearity in the restoring force $[6,8,9,12]$. Numerical simulations are then conducted in order to investigate the effect of the SMA on the LCO. In particular, it is shown that for certain parameter range, the SMA spring can lead to a significant decrease of the amplitude of the LCO.

\section{2DOFs Airfoil Model}

\subsection{Linear Model and Flutter Velocity}

The system under consideration is shown in Fig. 1. A Lagrangian formulation is used to express the evolution of the altitude $h$ (heave) and the angle of attack $\alpha$ (pitch) [5]. The kinetic energy reads $\mathcal{T}=\frac{1}{2} m \dot{h}^{2}+\frac{1}{2} I_{\alpha} \dot{\alpha}^{2}+S_{\alpha} \dot{h} \dot{\alpha}$, and the potential energy reads $\mathcal{V}=\frac{1}{2} k_{\alpha} \alpha^{2}+\frac{1}{2} k_{h} h^{2}$. In these expressions, $I_{\alpha}$ is the inertia moment and $S_{\alpha}$ the static moment which is directly related to the position of the airfoil gravity center and is the cause of the coupling. The source terms $F$ and $M$ (aerodynamic force and moment) are classically derived from the lift coefficient $C_{L}$ such as $F=\frac{1}{2} \rho U^{2} S C_{L}$ and $M=e F$, where $\rho$ is the fluid density, $U$ the upstream airspeed and $S$ the airfoil section. We assume that the angle of attack remains small, so that the lift coefficient depends linearly on $\alpha_{a p p}$ the apparent angle of attack, so that $C_{L}=\left(\partial C_{L} / \partial \alpha\right) \alpha_{a p p}$ $\left(\left.C_{L}\right|_{\alpha=0}=0\right.$ because the airfoil is symmetric). A pseudo-static approach is used, 

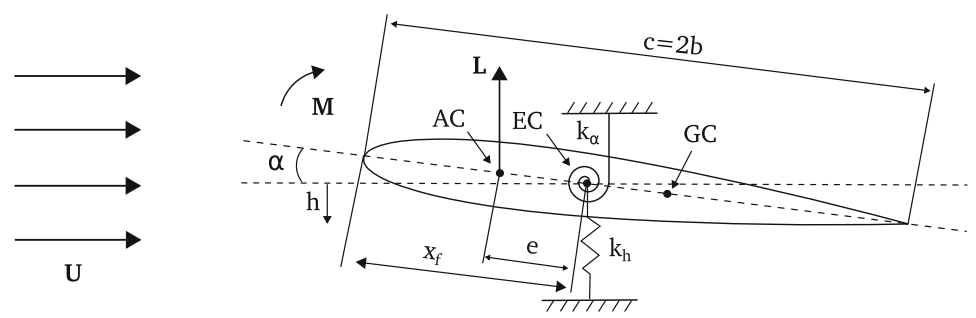

Fig. 1 Sketch of the airfoil model as a rigid body in uniform flow U with two dof: pitch $(\alpha)$ and heave $(h)$

i.e. the airfoil speed $\dot{h}$ is not neglected as compared to the upstream airspeed $U$ and $\alpha_{\text {app }}=\alpha+\dot{h} / U[5]$.

Lagrange equations lead to the following dynamical system:

$$
\begin{gathered}
{\left[\begin{array}{cc}
m & S_{\alpha} \\
S_{\alpha} & I_{\alpha}
\end{array}\right]\left[\begin{array}{c}
\ddot{h} \\
\ddot{\alpha}
\end{array}\right]+\left[\begin{array}{cc}
\frac{1}{2} \rho U S C_{L, \alpha} & 0 \\
-\frac{1}{2} e \rho U S C_{L, \alpha} & 0
\end{array}\right]\left[\begin{array}{c}
\dot{h} \\
\dot{\alpha}
\end{array}\right]} \\
+\left[\begin{array}{cc}
K_{h} & \frac{1}{2} \rho U^{2} S C_{L, \alpha} \\
0 & K_{\alpha}-\frac{1}{2} e \rho U^{2} S C_{L, \alpha}
\end{array}\right]\left[\begin{array}{c}
h \\
\alpha
\end{array}\right]=0 .
\end{gathered}
$$

In nondimensional form (1) reads

$$
\begin{aligned}
& {\left[\begin{array}{cc}
1 & x_{\alpha} \\
x_{\alpha} & r_{\alpha}^{2}
\end{array}\right]\left[\begin{array}{l}
y^{\prime \prime} \\
\alpha^{\prime \prime}
\end{array}\right]+\left[\begin{array}{cc}
\mu C_{L, \alpha} \Theta & 0 \\
-\mu \gamma C_{L, \alpha} \Theta & 0
\end{array}\right]\left[\begin{array}{c}
y^{\prime} \\
\alpha^{\prime}
\end{array}\right]} \\
& +\left[\begin{array}{cc}
\Omega^{2} & \mu C_{L, \alpha} \Theta^{2} \\
0 & r_{\alpha}^{2}-\mu \gamma C_{L, \alpha} \Theta^{2}
\end{array}\right]\left[\begin{array}{c}
y \\
\alpha
\end{array}\right]=0, \\
& y=\frac{h}{b}, \quad \tau=\frac{K_{\alpha} t}{I_{\alpha}}, \quad()^{\prime}=\frac{d}{d \tau}, \\
& r_{\alpha}=\sqrt{\frac{I_{\alpha}}{m b^{2}}}, \quad \mu=\frac{\rho b S}{2 m}, \quad x_{\alpha}=\frac{S_{\alpha}}{m b}, \\
& \Theta=\frac{U}{b} \sqrt{\frac{I_{\alpha}}{K_{\alpha}}}, \quad \Omega=\sqrt{\frac{K_{h} I_{\alpha}}{m K_{\alpha}}}, \quad \gamma=\frac{e}{b} .
\end{aligned}
$$

In order to gain insight on the critical parameter values, the flutter speed $\Theta_{f}$ can be derived analytically. Indeed, assuming that the airfoil motion is harmonic, $y=\Re(\tilde{y} \exp (p t))$ and $\alpha=\Re(\tilde{\alpha} \exp (p t))$ with $(p, \tilde{\alpha}, \tilde{y}) \in \mathcal{C}$, replacing $\alpha$ and $y$ by their new expressions in (2) it yields 
Fig. 2 Real (solid lines) and imaginary (dotted lines) parts of the solutions of $\operatorname{det}(\mathbf{A})=0$, with $\mathbf{A}$ defined in (3). Parameters of the problem are listed in Table 1

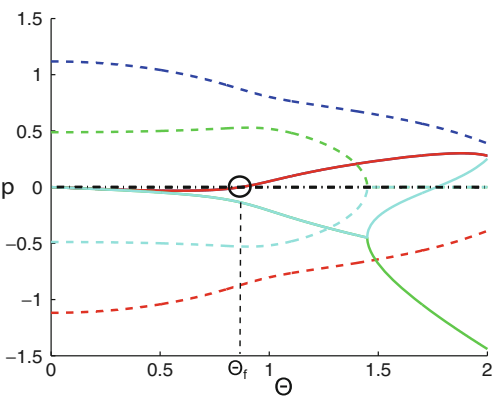

$\mathbf{A}\left[\begin{array}{l}\tilde{y} \\ \tilde{\alpha}\end{array}\right]=0$, with

Equation (3) has non trivial solutions $p_{i}$ when $\operatorname{det}(\mathbf{A})=0$. Using the parameter values of Table 1, the solutions $p_{i}$ versus the flow speed $\Theta$ is plotted in Fig. 2. At the flutter velocity $\Theta_{f}$, the real part of one of the solutions $p_{i}$ vanishes. Assuming then $p$ purely imaginary, separating real and imaginary parts in the equation $\operatorname{det}(\mathbf{A})=0$, and grouping the terms to eliminate $p$, the following expression is found for which $\Theta_{f}$ is solution

$$
\begin{aligned}
& \left(A_{1} \Theta^{2}-A_{2}\right)\left(A_{3} \Theta^{2}+A_{4}\right)=0, \\
& \text { with }\left\{\begin{array}{l}
A_{1}=\mu C_{L, \alpha}\left(r_{\alpha}^{2}+\gamma x_{\alpha}\right) \\
A_{2}=r_{\alpha}^{2} x_{\alpha}
\end{array}\right. \\
& \text { thus } \Theta_{f}=\sqrt{\frac{A_{2}}{A_{1}}}=\sqrt{\frac{r_{\alpha}^{2} x_{\alpha}}{\mu C_{L, \alpha}\left(r_{\alpha}^{2}+\gamma x_{\alpha}\right)}} .
\end{aligned}
$$

Using values of Table 1 we find $\Theta_{f}=0.87$.

\subsection{SMA Model}

The SMA spring is assumed to have pseudo-elastic behaviour, which is briefly recalled in Fig. 3. This nonlinear behaviour is characterized by a solid-solid phase change between two different states. The first one called austenite is stable at large 
Fig. 3 Pseudo-elastic behaviour of SMA and its microstructure for each phase

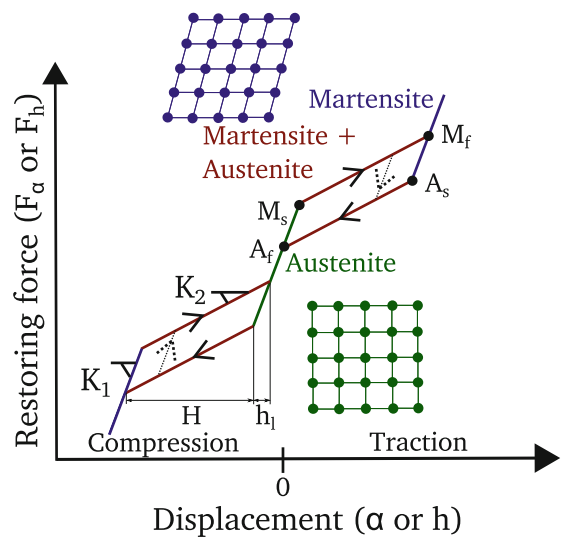

temperatures and is the natural state of the spring at rest. The microstructure of the austenite phase is also sketched in Fig. 3. When deformed, the microstructure of the SMA turns into a new phase called martensite, energetically stable at small temperatures and for which the microstructure is now oriented. The points in the stress-strain space where the transformations start and finish are usually denoted with the subscripts $s$ (start) and $f$ (finish), so that for instance $M_{f}$ refers to the point where the martensitic transformation has been fully accomplished in the SMA structure.

The path followed in the stress-strain relationship is not the same when the material is loaded or unloaded, thus an hysteresis loop appears as illustrated in Fig. 3. This hysteretic phenomena is the most salient feature of the pseudo-elastic behaviour of SMA. The dissipated energy during a cycle is proportional to the area of the hysteresis loop so that the more the loop area is large, the more energy is prone to be dissipated in the device.

The nonlinear behaviour of single dof SMAs can be derived from a general, threedimensional model inferred from thermodynamical laws and then reduced by considering ad-hoc assumptions, see e.g. [2, 7, 10, 11]. In this case the model contains, in-built within the oscillator equation, additional equations governing the evolution of the fraction of martensite, the description of heat transfer, and the thermodynamic force, which expression is derived from a pseudopotential of dissipation that can include yields functions in order to express the phase transformations [11]. These modeling features are typical of hysteretic systems [15] and give rise to a complex formulation which, in turn, induces numerical difficulties for solving the whole system. Contrary to this general approach, we use in this contribution a simple heuristic model instead, as it has the capacity to retrieve the main features of the dynamical behaviour within a light computational framework. It is built following the sketch in Fig. 3 by approximating each part of the diagram by a linear relationship, $h_{l}$ modelize the beginning of the martensitic transformation and $H$ the end of the martensitic transformation. An internal auxiliary variable playing the role of the fraction of martensite is defined so as to keep the memory of the precedent state of the material in a dynamical simulation. For simplification, it is assumed that the slopes of 
Table 1 Nondimensional

aeroelastic parameters

\begin{tabular}{l|l|l|l|l|l}
\hline$r_{\alpha}$ & $\mu$ & $x_{\alpha}$ & $\Omega$ & $\gamma$ & $C_{L, \alpha}$ \\
\hline 0.5 & $1 / 10 \pi$ & 0.2 & 0.5 & 0.4 & $2 \pi$ \\
\hline
\end{tabular}

the purely austenitic and purely martensitic phases are equal, as well as the slopes during the reverse or transverse transformations, so that the stiffness of the SMA are defined by $K_{1}$ and $K_{2}$, only as shown in Fig. 3. It is also assumed that the behaviour of the spring is symmetric in traction and compression. Internal loops are described following the sketch in Fig. 3 and we call $A_{S M A}$ the area of the maximal internal loop. The area $A_{S M A}$ is completely defined by the four parameters $K_{1}, K_{2}, h_{l}$ and $H$ with the following relationship

$$
A_{S M A}=h_{l} H \sqrt{1+K_{1}^{2}} \sqrt{1+K_{2}^{2}} \cos \left(\tan ^{-1}\left(1 / K_{1}\right)+\tan ^{-1}\left(K_{2}\right)\right) .
$$

For the remainder of the study, the four parameters that will be considered are $A_{S M A}$, $K_{1}, K_{2}$ and $h_{l}\left(A_{S M A}\right.$ instead of $H$ as it is more physically meaningful).

After the flutter instability, with the physical parameters from Table 1 the wing experiences large-amplitude motions especially on the pitch mode, whereas the amplitude of the motion of the heave mode remains fairly small. In order to take advantage of the dissipative properties of the SMA, it appears logical to include a SMA spring on the torsional motion, whereas the flexural spring is left unchanged with a linear behaviour law.

\subsection{Final Model}

To ensure a LCO after bifurcation occurs (no matter using SMA or not) a cubic stiffness is added on both modes ( $\xi_{y}$ for heave and $\xi_{\alpha}$ for pitch). The final model with a SMA torsional spring is then derived by replacing $r_{\alpha}^{2} \alpha$ from (2) with the nonlinear behaviour of the SMA spring $f_{N L}^{S M A}$ depicted in Fig. 3. It reads:

$$
\begin{gathered}
{\left[\begin{array}{cc}
1 & x_{\alpha} \\
x_{\alpha} & r_{\alpha}^{2}
\end{array}\right]\left[\begin{array}{l}
y^{\prime \prime} \\
\alpha^{\prime \prime}
\end{array}\right]+\left[\begin{array}{cc}
\mu C_{L, \alpha} \Theta & 0 \\
-\mu \gamma C_{L, \alpha} \Theta & 0
\end{array}\right]\left[\begin{array}{c}
y^{\prime} \\
\alpha^{\prime}
\end{array}\right]+} \\
{\left[\begin{array}{cc}
\Omega^{2} & \mu C_{L, \alpha} \Theta^{2} \\
0 & -\mu \gamma C_{L, \alpha} \Theta^{2}
\end{array}\right]\left[\begin{array}{c}
y \\
\alpha
\end{array}\right]=\left[\begin{array}{c}
-\xi_{y} y^{3} \\
-f_{N L}^{S M A}(\alpha)-\xi_{\alpha} \alpha^{3}
\end{array}\right] .}
\end{gathered}
$$




\section{Results and Discussion}

The aeroelastic parameters for the two-dofs system have been selected according to [5], they are listed in Table 1. Equation (5) is integrated in time with a fourthorder Runge-Kutta scheme. The initial condition is generally prescribed as a small perturbation on the heave mode. The results shown in Fig. 4 are made for $\xi_{y}=\xi_{\alpha}=0$, thus the nonlinearity is exclusively due to the SMA.

When $\Theta<\Theta_{f}$, the airfoil motion decreases and tends to zero, see Fig. 4a. When $\Theta \geq \Theta_{f}$, the flutter instability occurs and the position at rest is not stable anymore. However, the energy of the LCO can be dissipated by the SMA, so that for a certain range of reduced velocity, the amplitude of the motion saturates thanks to the nonlinear behaviour of the SMA, as illustrated in Fig. 4b. When the motion amplitudes of the LCO are beyond the end of the martensitic transformation, the potential of dissipation of the SMA is reached, so that divergent motions are retrieved. The critical speed above which the motion diverges is denoted $\Theta_{c}$. The first observed and awaited effect of adding the SMA is to increase $\Theta_{c}$ thanks to the damping property of the hysteresis loop, an intermediate stage where LCO with small amplitude is present. In a first step the energy evolution is investigated to ensure the LCO is entirely due to the hysteresis loop of the SMA and in a second step, a parametric study of the SMA is made to understand its influence on the LCO.

Fig. 4 Effect of the SMA on the linear flutter instability $\left(\xi_{y}=\xi_{\alpha}=0\right)$ for three different reduced velocities: a Linear stability case, $\Theta=0.86$ b Instability case, with LCO due to SMA dissipation, $\Theta=0.91$ c Instability case where SMA cannot dissipate enough energy, and an exponential growth is observed,

$\Theta=0.93\left(\Theta_{f}=0.87\right.$ and $\left.\Theta_{c}=0.926\right)$. Blue heave motion $y$, green pitch $\alpha$. The curves are normalized to their maximal value
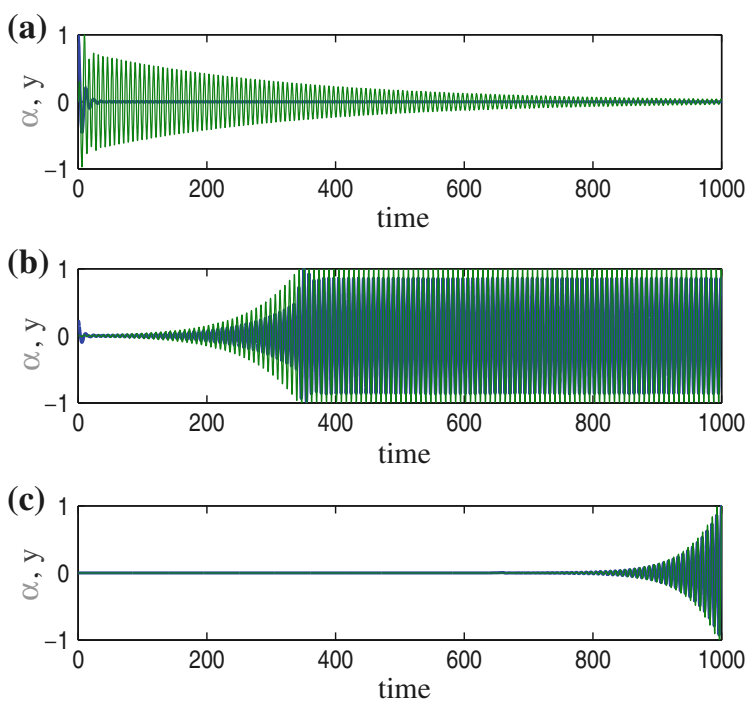
Fig. 5 Total energy $e_{t o t}$ versus time for the aeroelastic system (parameters of Table 1), with and without SMA, and for $\Theta=0.9$

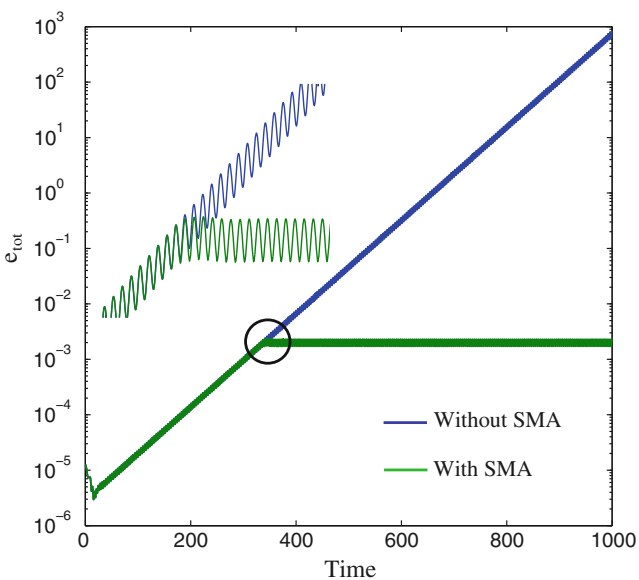

\subsection{Energy Exchange}

We investigate the energy evolution with time for the case $\Theta_{f}<\Theta<\Theta_{c}$. The energy contained in the airfoil reads

$$
E_{\text {tot }}=\mathcal{T}+\mathcal{V}=\frac{1}{2}\left(m \dot{h}^{2}+I_{\alpha} \dot{\alpha}^{2}+K_{\alpha} \alpha^{2}+K_{h} h^{2}\right)+S_{\alpha} \dot{h} \dot{\alpha}
$$

Then the nondimensional energy is

$$
e_{t o t}=\frac{1}{2}\left(\dot{y}^{2}+r_{\alpha}^{2} \dot{\alpha}^{2}+k_{\alpha} \alpha^{2}+\Omega^{2} y^{2}\right)+x_{\alpha} \dot{y} \dot{\alpha}
$$

Its evolution is plotted in Fig. 5 for system with and without SMA. For each cycle the energy increase of the system without SMA corresponds exactly to the energy dissipated by the SMA in its internal loop (for example right after the system with SMA enters in its first internal loop this loop dissipates $1.19810^{-4}$ and during this time the system without SMA grew $1.18910^{-4}$ ). Hence in this regime, the energy saturates to a finite value. This clearly shows that the LCO appearing when $\Theta_{f}<$ $\Theta<\Theta_{c}$ is entirely due to the SMA hysteresis loop. We now explore the influence of the SMA parameters on the LCO.

\subsection{Influence of SMA Parameters}

The global effect of the SMA parameters on the behaviour of the flutter instability is studied through the bifurcation diagram for varying flow velocities $\Theta$. Fig. 6 shows such a bifurcation diagram for a given SMA spring (the amplitude of pitch motion 
Fig. 6 Bifurcation diagram of the 2 dof aeroelastic system with cubic stiffness $\left(\xi_{y}=\xi_{\alpha}=1\right)$. Black Without SMA spring. Red With SMA spring (selected values: $h_{l}=0.05$,

$K_{1}=10 K_{2}$ and $A_{S M A}=2.35 \times 10^{-3}$ )

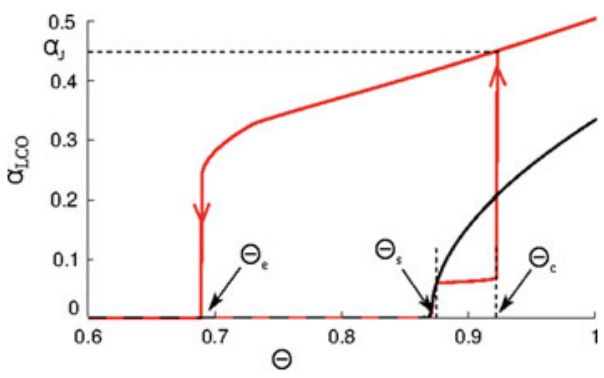

LCO is represented versus $\Theta$ ). The diagram is obtained numerically by direct integration in time of (5). The flow velocity is increased step by step, one time forward and one time backward, in order to get all the solution branches. When changing $\Theta$ to $\Theta+\delta \Theta$, the initial condition for $\Theta+\delta \Theta$ is selected as the steady state of the previous simulation launched for $\Theta$. The black line in Fig. 6 shows the bifurcation diagram when only a cubic nonlinear restoring force is considered, no SMA is added to the system. In this case the classical supercritical bifurcation is retrieved. The diagram with a given SMA is represented with the red line.

The first striking feature one can observe is that due to the softening effect brought by the SMA, the bifurcation is now subcritical. The gain in using enhanced damping properties thanks to the hysteresis loop is illustrated through the appearance of the points denoted $\Theta_{s}$ (flow velocity for which the oscillations enters the loop and thus the enhanced damping capacity is present) and $\Theta_{c}$ (the point for which the martensitic transformation is finished so that the damping capacity has been fully exploited). Above $\Theta_{c}$, a jump is observed to a branch parameterized by $\alpha_{J}$ (amplitude at $\Theta_{c}$ ) where the LCO amplitudes are larger than without SMA. Hence the advantage of the enhanced damping capacity is here completely lost due to the appearance of this subcritical branch where large-amplitude motions are observed. Finally, the point where this subcritical branch disappears when decreasing the flow velocity, denoted $\Theta_{e}$, defines a dangerous range of flow velocities where the system could jump to the higher branch.

The SMA parameters can be optimized in order to fulfill the following targets:

- decrease the amplitude $\alpha_{J}$ of the secondary branch as much as possible. In the best case, avoid subcriticality,

- decrease $\Theta_{s}$ so as to bring this point as close as possible to the flutter velocity,

- increase $\Theta_{c}$ as much as possible so as to take full advantage of the enhanced damping capacity of the SMA,

- increase $\Theta_{e}$ so as to avoid the large range of subcriticality where the two solution branches coexist.

Numerous simulations have been done to obtain the best parameters $h_{l}$ and $K_{1} / K_{2}$, in order to fulfill at best these objectives. Figure 7c sums up some of the results obtained for illustration. One can conclude that in order to decrease the value $\alpha_{J}$ describing the upper branch, one has to select a large value for $h_{l}$, and in this case 
Fig. 7 a LCO amplitude versus flow speed for different SMA parameters $\left(\xi_{h}=\xi_{\alpha}=1\right.$ and $A_{S M A}$ constant). b Corresponding restoring force for $\Theta=\Theta_{c}$ on the left $h_{l}=0.05$ and $K_{1} / K_{2}=10$ and on the right $h_{l}=0.12$ and $K_{1} / K_{2}=10$ (a)

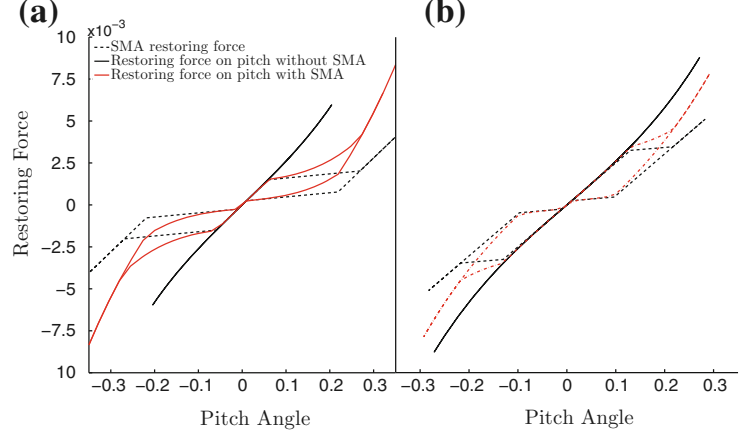

(c)

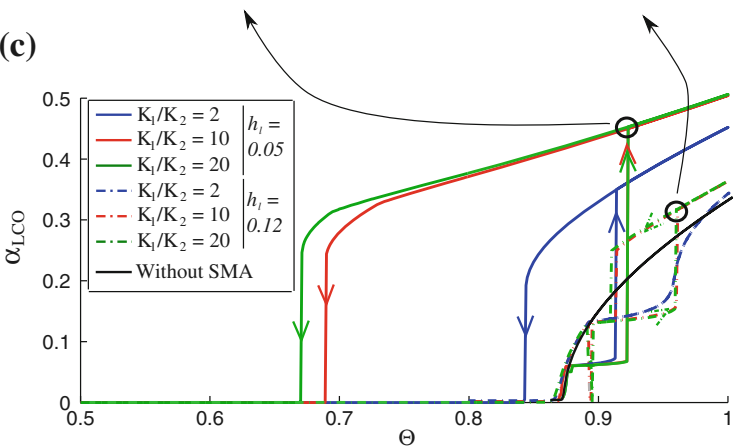

it appears then more appropriate to take smaller values for the ratio $K_{1} / K_{2}$. One can also observe the advantageous effect of using a small value for $K_{1} / K_{2}$ when $h_{l}$ has been selected small, as it increases substantially the value of $\Theta_{e}$. Table 2 summarizes the effect of the coefficients with respect to the targeted objectives relative to the different remarkable points $\Theta_{s}, \Theta_{e}, \Theta_{c}$ and $\alpha_{J}$. A simple plus/minus sign code is used for a quick understanding of the effect of each coefficients: a plus sign indicates an advantageous effect whereas a minus sign indicates a detrimental effect with respect to the target. From this table and Fig. $7 \mathrm{c}$ it appears clear that the best choice is a large $h_{l}$ together with a small ratio $K_{1} / K_{2}$.

Figure $7 \mathrm{a}, \mathrm{b}$ show the restoring force at two particular points in order to get physical insight in the results obtained. In Fig. 7a, a disadvantageous case is selected and one can observe that the softening effect is the dominating feature of the nonlinear restoring force of the SMA, explaining the enforced subcriticality observed on the bifurcation diagram. This adverse case helps also in understanding that increasing blindly the hysteresis loop area $A_{S M A}$ is not a solution as it may have no effect on the subcriticality. When $K_{2}$ is too small, the sudden jump in amplitude observed for a small variation of force is detrimental as the system jumps to large amplitude motions and the energy of this vibratory state is too important. Figure $7 \mathrm{~b}$ illustrates an advantageous case where the softening effect is not too important and thus leads to an improved global behaviour in the bifurcation diagram. 
Table 2 Influence of the SMA constants on the identified points in the bifurcation diagram

\begin{tabular}{l|l|l|l|l}
\hline SMA parameters & \multicolumn{2}{l}{$K_{1} / K_{2}$} & \multicolumn{2}{l}{$h_{l}$} \\
\hline Bif. pt & Small & Large & Small & Large \\
\hline$\Theta_{s}$ & $=$ & $=$ & +++ & --- \\
\hline$\Theta_{e}$ & +++ & --- & --- & +++ \\
\hline$\Theta_{c}$ & - & + & --- & +++ \\
\hline$\alpha_{J}$ & ++ & -- & --- & +++ \\
\hline A & & & + & +
\end{tabular}

A plus sign shows an advantageous effect, whereas a minus sign indicates a detrimental effect on the targeted behaviour, while an equal sign shows no effect. The number of the sign is proportional to the magnitude of the effect

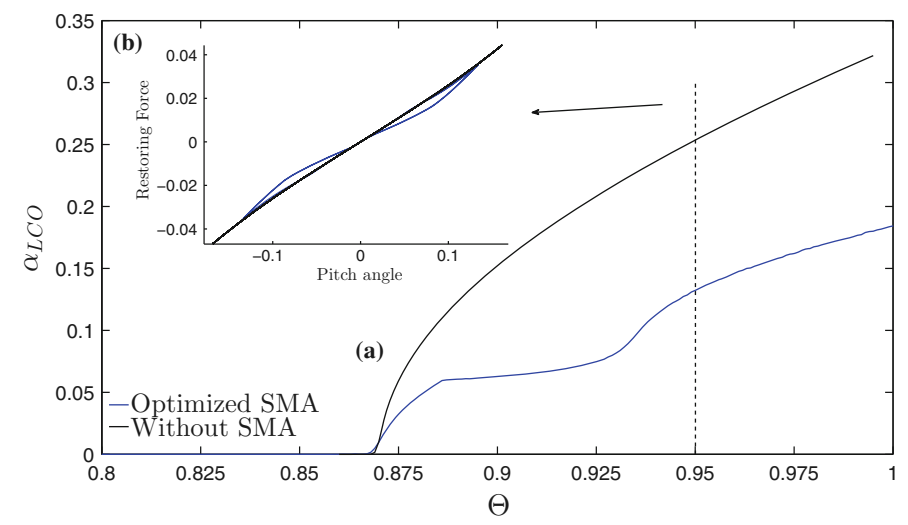

Fig. 8 a Bifurcation diagram with $h_{l}=0.05, K_{1} / K_{2}=1.5$ and $\xi_{\alpha}=3.4$. b Restoring force at $\Theta=0.95$

This analysis shows undoubtedly that the best choice would be to have both the case of the hysteresis loop together with a stronger hardening effect at very large amplitude. To fulfill these requests, the full martensite branch should have a hardening behaviour stronger that the one of the reference case (without SMA). Such a configuration is obtained for example with $h_{l}=0.05, K_{1} / K_{2}=1.5$ and $\xi_{\alpha}=3.4$. Figure 8 shows the bifurcation diagram obtained in this case. One can observe that supercriticality is enforced. Secondly a large range of small amplitudes of LCOs are observed between 0.875 and 0.93 , where the enhanced damping capacity of the SMA plays its role. Finally the improved hardening effect at large amplitude gives rise to a branch where amplitudes of LCOs are smaller, even when the transformation has been completed. 


\section{Conclusion}

Whether one wants to harvest energy or avoid devastating intabilities, the control of aeroelastic flutter is a critical issue. An option is discussed here by using SMA springs in pseudo-elastic behaviour, in order to use the potential of dissipation of such materials. A numerical study with a simple heuristic model for the behaviour of the SMA has shown that the amplitude of the LCO after the flutter velocity can be significantly reduced by adding a SMA spring on the pitch mode of the twodofs aeroelastic system. More precisely, the study of the bifurcation diagram clearly exhibits an advantageous effect of using the enhanced damping capacity of the SMA with the appearance of a branch of small amplitudes LCOs. However a detrimental effect appears because of the softening effect created by the solid-solid phase change. The optimized case is obtained by hardening the stiffness of the martensite phase. In this case it has been shown that subcriticality together with small amplitudes LCOs can be obtained.

The next steps of this research is to confront these preliminary findings with experiments. Complicating effects such as the dependence of the hysteresis loop on frequency, asymmetry of the SMA restoring force, aeroelastic nonlinearities due to stall phenomenon, will be studied and included in the model in order to obtain a global picture of the passive control of the flutter instability with SMA springs.

Acknowledgments This work is supported by Direction Générale de l'Armement (DGA) from French Ministry of Defense.

\section{References}

1. Bernardini, D., Rega, G.: The influence of model parameters and of the thermomechanical coupling on the behavior of shape memory devices. Int. J. Non-Linear Mech. 45(10), 933-946 (2010)

2. Bernardini, D., Vestroni, F.: Non-isothermal oscillations of pseudoelastic devices. Int. J. NonLinear Mech. 38(9), 1297-1313 (2003)

3. Bisplinghoff, R.L., Ashley, H., Halfman, R.L.: Aeroelasticity. Courier Dover Publications, New York (2013)

4. Doaré, O., Sbarra, A., Touzé, C., Moussa, M.O., Moumni, Z.: Experimental analysis of the quasistatic and dynamic torsional behaviour of shape memory alloys. Int. J. Solids Struct. (2011)

5. Dowell, E.H. et al. A Modern Course in Aeroelasticity. Kluwer Academic, Dordrecht (1994)

6. Ko, J., Strganac, T.W., Kurdila, A.J.: Adaptive feedback linearization for the control of a typical wing section with structural nonlinearity. Nonlinear Dyn. 18(3), 289-301 (1999)

7. Lacarbonara, W., Bernardini, D., Vestroni, F.: Nonlinear thermomechanical oscillations of shape-memory devices. Int. J. Solids Struct. 41(5-6), 1209-1234 (2004)

8. Lee, Y.S., Kerschen, G., McFarland, D.M., Hill, W.J., Nichkawde, C., Strganac, T.W., Bergman, L.A., Vakakis, A.F.: Suppressing aeroelastic instability using broadband passive targeted energy transfers, part 2: experiments. AIAA J. 45(10), 2391-2400 (2007)

9. Lee, Y.S., Vakakis, A., Bergman, L., McFarland, D.M., Kerschen, G.: Suppression aeroelastic instability using broadband passive targeted energy transfers, part 1: Theory. AIAA J. 45(3), 693-711 (2007) 
10. Moussa, M.O.: Modélisation du comportement dynamique non-linéaire des structures en matériaux à mémoire de forme. $\mathrm{PhD}$ thesis, Ecole Polytechnique (2012)

11. Moussa, M.O., Moumni, Z., Doaré, O., Touzé, C., Zaki, W.: Non-linear dynamic thermomechanical behaviour of shape memory alloys. J. Intell. Mater. Syst. Struct. 23(14), 1593-1611 (2012)

12. Platanitis, G., Strganac, T.W.: Control of a nonlinear wing section using leading-and trailingedge surfaces. J. Guidance Control Dyn. 27(1), 52-58 (2004)

13. Saadat, S., Salichs, J., Noori, M., Hou, Z., Davoodi, H., Bar-On, I., Suzuki, Y., Masuda, A.: An overview of vibration and seismic applications of niti shape memory alloys. Smart Mater. Struct. (2002)

14. Strelec, J.K., Lagoudas, D.C., Kahn, M.A., Yen, J.: Design and implementation of a shape memory alloy actuated reconfigurable airfoil. J. Intell. Mater. Syst. Struct. (2003)

15. Visintin, A.: Differential Models of Hysteresis, vol. 1. Springer, Berlin (1994) 